\title{
Editorial
}

\section{Comentario editorial sobre Laparo-endoscopia por acceso único: experiencia inicial}

\author{
En referencia al trabajo de este número que aparece en las páginas 172-181
}

\author{
Estevao Lima
}

Serviço de Urologia do Centro Hospital do Porto - Hospital Geral de Santo António, Porto, Portugal. Instituto de Ciências da Vida e da Saúde (ICVS), Escola de Ciências da Saúde da Universidade do Minho, Braga, Portugal

$\mathrm{D}$ esde la introducción de la cirugía vídeoasistida en la década de los ochenta, se ha demostrado que la reducción de las incisiones quirúrgicas diminuye el dolor y el tiempo de recuperación pos-operatorios. Kalloo et al., con el objetivo de realizar cirugías abdominales sin cicatrices, realizó en 2004, el abordaje de la cavidad peritoneal por vía transgástrica ${ }^{1}$. Fue el nacimiento del concepto de NOTES (Natural Orifice Transluminal Endoscopic Surgery). La Cirugía endoscópica transluminal por orificios naturales, además de evitar las incisiones abdominales y todas las consecuencias de las mismas, tales como hernias y infecciones de las heridas operatorias, tendría el teórico potencial de disminuir o evitar el dolor y el tiempo de recuperación en el pos-operatorio. De hecho, el estímulo del dolor visceral es diferente del dolor somático y teóricamente las incisiones en las vísceras no causan dolor ${ }^{2}$.

A pesar de todas las potenciales ventajas del abordaje transgástrico, los trabajos experimentales demostraron que esta vía presenta varias limitaciones de orden quirúrgica y complicaciones graves. Las limitaciones quirúrgicas de esta vía se deben a la utilización de endoscopios flexibles con canales de trabajo paralelos e instrumentos flexibles. Las potenciales complicaciones se relacionan con el hecho de no ser estéril esta vía y ser de difícil encerramiento, pudiendo llevar a la contaminación peritoneal. De hecho, hasta hoy en día, todos los métodos de encerramiento gástrico endoscópico se mostraron de difícil ejecución y no totalmente seguros y eficaces $^{2}$.

Como consecuencia, otros abordajes de la cavidad abdominal han sido descritas, tales como: 1) la vía transcolónica, que mantiene muchas de las limitaciones previamente descritas en la vía transgástrica tales como, no ser estéril y la inexistencia de mecanismos de encerramiento de la colotomía seguros y eficaces; 2) la vía transvesical que no tiene las limitaciones previamente descritas en la vía transgástrica y transcolónica porque de hecho es naturalmente estéril y 3) la vía transvaginal ${ }^{2,3}$. Este último abordaje de la cavidad peritoneal, a pesar de haber sido ya utilizada en el pasado por muchos ginecólogos para el diagnóstico y tratamiento de patologías pélvicas, recientemente se ha vuelto muy popular como puerta de acceso abdominal para NOTES. De hecho, es la única puerta utilizada por rutina en humanos, lo que se debe a su fácil esterilización y al hecho de ser la única en que existe un método de encerramiento comprobadamente simple y seguro. Tiene, sin embargo, como limitaciones, el estar disponible solamente en el sexo femenino y cuando se utiliza de una forma aislada, no permite la realización de cirugías más complejas como es el caso de la nefrectomía. Así, para la realización de nefrectomías se necesita colocar dos puertas transabdominales adicionales (técnica híbrida) ${ }^{2}$.

Rene Sotelo et al. presentan un articulo con 28 procedimientos usando el "Single Port Access" (SPA) o la "Single Incision Laparoscopic Surgery" (SILS) o mas recientemente denominado, después de una reunión de consenso como "Laparo-endoscopic Single Site Surgery” (LESS Surgery). Este concepto surge como una tentativa, con las condiciones tecnológicas actuales, de realizar de una forma segura, cirugía sin cicatrices utilizando como puerta de acceso el ombligo. En verdad, la colocación de una puerta transabdominal hace posible la realización de cirugías complejas que por NOTES puro serian demasiado audaces, es decir, no completamente ausente de riesgo quirúrgico. Recientemente, han sido descritos varios procedimientos quirúrgicos usando el concepto de "LESS Surgery" tales como nefrectomías simples y radicales, nefrectomía del 
donante vivo, pieloplastias, nefrectomías parciales, criocirugía de tumores renales, ureterolitotomía, varicocelectomías, sacrocolpopexías, prostatectomías simples y hasta cistectomías y prostatectomías radicales $^{4-7}$. Los autores de este articulo deben ser felicitados por sus resultados ya que describen muchas de estas técnicas y ser mismo los pioneros en la enterocistoplastía de aumento y co-pioneros en la prostatectomía transvesical simple y radical ${ }^{5,6}$. Esta última cirugía a pesar de estar muy bien proyectada podrá no ser posible de aplicarla en las situaciones de vejiga muy espesada y de baja capacidad.

A pesar haber sido descritas muchas técnicas de "LESS Surgery" sin complicaciones, se debe señalar determinados aspectos particulares. En este tipo de abordaje se utiliza un trocar único especial (R-Port, Advanced Surgical Concepts, Dublin, Ireland o UniX, (Pnavel Systems, Cleveland, USA) con 3 a 4 puertas por donde se insertan en la cavidad abdominal instrumentos articulados o doblados desarrollados específicamente para esta técnica quirúrgica (Real Hand, Novare Surgical Systems, Cupertino, USA, and Autonomy Laparo-angle, Cambridge Endo, Framingham, USA), intentando superar las dificultades de falta de triangulación, dificultad en la retracción de los tejidos y choque entre los instrumentos ${ }^{4}$. También para evitar este último aspecto, los instrumentos de "LESS Surgery" son mas largos que los de la laparoscopia convencional y las cámaras del tipo "EndoEye" de preferencia de $5 \mathrm{~mm}$ y con punta flexible. Este tipo de cirugía requiere alguna curva de aprendizaje y coordinación con un ayudante de cámara, con necesidad de entrenar en animales de laboratorio. Otro aspecto negativo a referir es la contra-indicación de la colocación de un trocar a nivel umbilical en pacientes obesos, altos o con abdomen muy amplio. A parte de eso, la colocación de un trocar fuera del ombligo como en la punta de la $12^{\text {a }}$ costilla o infra-umbilical, como ocurrió en la prostatectomía transvesical simple descrita por Rene Sotelo et al., conduce a una perdida del aspecto estético.
De este modo este tipo de abordaje quirúrgico necesita futuros estudios comparativos con la laparoscopia convencional, la "needlescopy" (mini laparoscopia) para mostrar ventajas clínicamente significativas, ya que hasta el potencial beneficio estético permanece dudoso ${ }^{8}$. La "LESS Surgery" no parece representar una verdadera innovación tal como ocurrió en el pasado con el paso de cirugía abierta para cirugía laparoscópica; sin embargo, la cirugía endoscópica se puede beneficiar de los nuevos instrumentos quirúrgicos y algunas técnicas podrán tener su aplicación en ciertas situaciones clínicas.

\section{REFERENCIAS}

1. Kalloo AN, Singh VK, Jagannath SB, Niiyama H, Hill SL, Vaughn CA et al. Flexible transgastric peritoneoscopy: a novel approach to diagnostic and therapeutic interventions in the peritoneal cavity. Gastrointest Endosc. 2004;60:114-117.

2. Lima E, Rolanda C, Correia-Pinto J. Transvesical endoscopic peritoneoscopy: intra-abdominal scarless surgery for urologic applications. Curr Urol Rep. 2008;9:50-54.

3. Lima E, Rolanda C, Pêgo JM, Henriques-Coelho T, Silva D, Carvalho JL et al. Transvesical endoscopic peritoneoscopy: a novel $5 \mathrm{~mm}$ port for intra-abdominal scarless surgery. J Urol. 2006; 176:802-805.

4. Canes D, Desai MM, Aron M, Haber GP, Goel RK, Stein RJ et al. Transumbilical single-port surgery: evolution and current status. Eur Urol. 2008;54:1020-1029.

5. Desai MM, Aron M, Canes D, Fareed K, Carmona O, Haber GP et al. Single-port transvesical simple prostatectomy: initial clinical report. Urology. 2008;72:960-965.

6. Desai MM, Aron M, Berger A, Canes D, Stein R, Haber GP et al. Transvesical robotic radical prostatectomy. BJU Int. 2008;102: 1666-1669.

7. Kaouk JH, Goel RK, Haber GP, Crouzet S, Desai MM et al. Single-port laparoscopic radical prostatectomy. Urology. 2008; 72:1190-1193.

8. Raman JD, Bagrodia A, Cadeddu JA. Single-Incision, Umbilica Laparoscopic versus Conventional Laparoscopic Nephrectomy: A Comparison of Perioperative Outcomes and Short-Term Measures of Convalescence. Eur Urol. 2008 Aug 13. [Epub ahead of print].

Correspondencia autor: Dr. Estevao Lima

Servicio de Urología.

Hospital de Oporto - Hospital Geral de Santo Antonio

Oporto. Portugal

E-mail autor: estevaolima@ecsaude.uminho.pt

Información artículo: Editorial

Trabajo recibido: enero 2009 